\title{
장oㅇ
}

\section{THE IM2004 INFORMATION MANAGEMENT AWARDS}

\section{Announcement of winners}

The ninth annual Information Management Awards were presented at a dinner at the Grosvenor House Hotel on Thursday 2nd December in front of an audience of over 1050 finalists, press and guests.

The Journal of Targeting, Measurement and Analysis for Marketing is the exclusive media partner for the Personalised Information Award. Dr Jonathan Reynolds, Oxford Institute of Retail Management and member of the Editorial Board of the journal, was a member of the judging panel, and the award was presented by John Ozimek, Managing Editor of the Journal of Database Marketing E Customer Strategy Management.

Seventeen original entries were submitted and Clever-Stuff won the award for their product disgo Xkey. The runner-up was KeyComputing and WCC Services won a Commendation. Clever-Stuff also won a Commendation in the Product of the Year Award for the overall best product of 2004.

The full list of winners and synopses of the winning entries can be viewed on http://im2004.elanconf.co.uk/winners.php

The Information Management Awards are the premier European recognition of excellence and innovation in the management of business information. They are a showcase for organisations that have demonstrated the vision and business skills to implement highly successful projects based on leading-edge technologies such as CRM, Business Intelligence, Intranet and Content Management, and for ground-breaking innovative products for Personalised Information, Information Security and Information Storage.

The 2004 Awards were presented at the Awards dinner at the Grosvenor
House Hotel on Thursday 2nd December in front of an audience of nearly 1050 finalists, press and guests. The host and entertainer for the evening was Barry Cryer, veteran of countless radio and television comedy shows.

From 17 original entries in the Personalised Information Product category, the Award for the best new product went to Clever-Stuff for their product disgo Xkey, presented by John Ozimek, Editor, Journal of Database Marketing \& Customer Strategy Management. The runner-up was KeyComputing and WCC Services won a Commendation. 
disgo Xkey is a pocketable USB device that enables employees to work from any PC, complete with the critical applications, corporate data and security needed for productive work. disgo Xkey thus enables office-based employees to extend their workday from home, without the physical and financial burden of a dedicated laptop. Users of disgo Xkey simply plug it into their home PCs, enter their passwords, and are then ready to access their complete messaging environment - e-mails, calendar and contacts - for immediate work online or offline. disgo Xkey also contains other corporate data, such as spreadsheets, presentations and documents, needed for work outside of the office.

The particular benefits are that it plugs into any computer and is ready for work, without the need of any pre-installed components on the client PC, or any server-side installation or configuration. It replicates employees' work environments, enabling them to be productive from their homes in cases of extreme weather, network disruption, server failure or other disasters.

It enables them to work on their calendars, e-mails and contacts on any computer regardless of what applications are installed, and it works in any network state: online, offline and behind firewalls, performing well even on slow or intermittent connections. It stores hundreds of megabytes of corporate data, such as presentations and spreadsheets. For security purposes, the information on disgo Xkey is encrypted in case the device is lost or stolen, and encrypts all communications to and from the Exchange server. It has an inbuilt mechanism to protect users from malicious spyware applications that monitor their work and removes traces of work inadvertently left behind.

This new device is no bigger than a lighter but incorporates a powerful microprocessor, a crypto engine for AES level data encryption, up to $2 \mathrm{~GB}$ of flash storage, a full Microsoft Exchange client, a database for securely storing Exchange data, a synchronisation engine that connects to the corporate Exchange server and an embedded SSL VPN security application.

According to studies by META Group (2002) and Gartner (Sept 2003), employees increase productivity, working an extra 2-6 hours a week, when equipped to work from home. disgo Xkey thus transforms a business from a 9 to 5, office-based company into a highly responsive $24 / 7$ location-independent business. The costs are such that disgo Xkey mobilises up to 20 people for the total cost-of-ownership of a single laptop, while weighing 0.5 per cent of a laptop so that employees can carry them everywhere.

Further information: www.mydisgo.com/xkey

For further information on the 2004 Information Management Awards, please contact Lisa Di Bernardo, by telephone on +44 (0)1707 373701 or e-maillisad@elanconf.co.uk 